\title{
Effects of long-term post-ischemic treadmill exercise on gliosis in the aged gerbil hippocampus induced by transient cerebral ischemia
}

\author{
JI HYEON AHN ${ }^{1 *}$, MYOUNG CHEOL SHIN ${ }^{2 *}$, JOON HA PARK ${ }^{3}$, IN HYE KIM $^{3}$, JEONG-HWI CHO ${ }^{3}$, \\ TAE-KYEONG LEE ${ }^{3}$, JAE-CHUL LEE ${ }^{3}$, BAI HUI CHEN ${ }^{4}$, BICH NA SHIN ${ }^{4}$, HYUN-JIN TAE $^{1}$, JINSEU PARK ${ }^{1}$, \\ SOO YOUNG CHOI ${ }^{1}$, YUN LYUL LEE ${ }^{4}$, DAE WON KIM ${ }^{5}$, YANG HEE KIM ${ }^{6}$, MOO-HO WON $^{3}$ and JUN HWI CHO ${ }^{2}$ \\ ${ }^{1}$ Department of Biomedical Science and Research Institute for Bioscience and Biotechnology, \\ Hallym University, Chuncheon, Gangwon 24252; Departments of ${ }^{2}$ Emergency Medicine and ${ }^{3}$ Neurobiology, \\ School of Medicine, Kangwon National University, Chuncheon, Gangwon $24341 ;{ }^{4}$ Department of \\ Physiology, College of Medicine, Hallym University, Chuncheon, Gangwon 24252; ${ }^{5}$ Department of \\ Biochemistry and Molecular Biology, and Research Institute of Oral Sciences, College of Dentistry, \\ Kangnung-Wonju National University, Gangneung, Gangwon 25457; ${ }^{6}$ Department of Surgery, \\ School of Medicine, Kangwon National University, Chuncheon, Gangwon 24341, Republic of Korea
}

Received August 14, 2016; Accepted March 2, 2017

DOI: $10.3892 / \mathrm{mmr} .2017 .6485$

\begin{abstract}
Therapeutic exercise is an integral component of the rehabilitation of patients who have suffered a stroke. The objective of the present study was to use immunohistochemistry to investigate the effects of post-ischemic exercise on neuronal damage or death and gliosis in the aged gerbil hippocampus following transient cerebral ischemia. Aged gerbils (male; age, 22-24 months) underwent ischemia and were subjected to treadmill exercise for 1 or 4 weeks. Neuronal death was detected in the stratum pyramidale of the hippocampal CA1 region and in the polymorphic layer of the dentate gyrus using cresyl violet and Fluoro-Jade B histofluorescence staining. No significant difference in neuronal death was identified following 1 or 4 weeks of post-ischemic treadmill exercise. However, post-ischemic treadmill exercise affected gliosis (the activation of astrocytes and microglia).
\end{abstract}

Correspondence to: Professor Moo-Ho Won, Department of Neurobiology, School of Medicine, Kangwon National University, 1 Kangwondaehak, Chuncheon, Gangwon 24341, Republic of Korea E-mail:mhwon@kangwon.ac.kr

Professor Jun Hwi Cho, Department of Emergency Medicine, School of Medicine, Kangwon National University, 1 Kangwondaehak, Chuncheon, Gangwon 24341, Republic of Korea

E-mail: cjhemd@kangwon.ac.kr

"Contributed equally

Key words: aging, ischemic stroke, post-ischemic treadmill exercise, pyramidal neurons, astrocytes, microglia
Glial fibrillary acidic protein-immunoreactive astrocytes and ionized calcium binding adaptor molecule 1-immunoreactive microglia were activated in the CA1 and polymorphic layer of the dentate gyrus of the group without treadmill exercise. Conversely, 4 weeks of treadmill exercise significantly alleviated ischemia-induced astrocyte and microglial activation; however, 1 week of treadmill exercise did not alleviate gliosis. These findings suggest that long-term post-ischemic treadmill exercise following transient cerebral ischemia does not influence neuronal protection; however, it may effectively alleviate transient cerebral ischemia-induced astrocyte and microglial activation in the aged hippocampus.

\section{Introduction}

Transient forebrain ischemia induces the damage/death of pyramidal neurons in the CA1 region of the hippocampus (1-3). It has previously been reported that aged animals are less vulnerable to ischemia, and ischemia-induced neuronal degeneration occurs much later than in adult animals (4-6).

Microglia, which are primary immune cells that are located in the central nervous system, and astrocytes, which act as important modulators of neuronal activity, are both involved in maintaining homeostasis of the brain microenvironment (7). Microglia and astrocytes maintain a resting phenotype under physiological conditions; however, in the process of aging or pathological conditions, including ischemia-reperfusion injury, they exhibit activation with morphological and functional alterations, including hypertrophy and the release of various factors, which have been reported to modulate the injury process (8-10). It is well known that glial cells serve complex roles in neuroinflammation and in the regeneration of brain tissue following ischemic insults $(11,12)$. 
In the case of stroke, exercise treatment has been used in humans to aid the remaining functions (13). In experimental animals, exercise reduces astrocyte and microglial activation in the acute phase following transient focal ischemia in rats (14) and traumatic brain injury in mice (15). The effects of exercise on glial activation in animal models of brain injuries have previously been investigated; however, long-term alterations to glial activation in the ischemic hippocampus in aged animals have yet to be fully elucidated. Therefore, the present study aimed to investigate the effects of post-ischemic exercise on neuronal damage and gliosis in the hippocampus following transient cerebral ischemia in the aged gerbil, a useful animal model for transient cerebral ischemia and aging research (16-19).

\section{Materials and methods}

Experimental animals. A total of 35 male Mongolian gerbils (Meriones unguiculatus; age, 22-24 months; weight, 80-90 g) were supplied by the Experimental Animal Center, Kangwon National University (Chuncheon, South Korea). Gebrils were housed in a conventional facility, at a temperature of $23 \pm 3^{\circ} \mathrm{C}$ and relative humidity of $55 \pm 5 \%$, under $12 / 12 \mathrm{~h}$ light/dark cycles, and were allowed free access to food and water. Animal handling and experimental protocols were approved by the Institutional Animal Care and Use Committee of Kangwon National University (approval no. KW-130424-1). The gerbils were randomly divided into five groups: i) Sham group $(n=7)$, which underwent sham surgery; ii) ischemia group $(n=7)$, which underwent 5 min of transient forebrain ischemia; iii) ischemia-SD4 group $(\mathrm{n}=7)$, which had a sedentary routine for 4 weeks (SD4) from 5 days post-ischemia; iv) ischemia-TR1 group $(n=7)$, which performed 1 week treadmill exercise (TR) from 5 days post-ischemia; and, v) ischemia-TR4 group ( $n=7)$, which performed 4 weeks TR from 5 days post-ischemia. The animals were sacrificed 31 days following ischemia; at which point, the TR training was concluded in the ischemia-TR4 group.

Induction of transient cerebral ischemia. Following the method described in our previous study (20), the gerbils were anesthetized with a mixture of $2.5 \%$ isoflurane (Baxter Healthcare Corporation, Deerfield, IL, USA) in 33\% oxygen and $67 \%$ nitrous oxide. After a sagittal ventral midline incision, common carotid arteries were carefully separated from the respective vagal nerves and were occluded for 5 min using nontraumatic aneurysm clips (Yasargil FE 723K; Aesculap AG, Tuttlingen, Germany). Following occlusion for $5 \mathrm{~min}$, the clips were removed and the wounds were sutured with wound clips (12022-09; Fine Science Tools, Inc., Foster City, CA, USA). Normothermic body (rectal) temperature $\left(37 \pm 0.5^{\circ} \mathrm{C}\right)$ was monitored until the animals completely recovered from anesthesia. Sham surgery animals were subjected to the same surgical procedures without the occlusion of the bilateral common carotid arteries.

Treadmill exercise. The running speed and duration of treadmill exercise was determined according to Sim's protocol (21-23), with modification. Briefly, from 5 days post-ischemia, the gerbils in the TR groups were forced to run on a motorized treadmill for $30 \mathrm{~min} /$ day and 5 days/week for 1 or 4 consecutive weeks. The exercise workload consisted of running at a speed of $5 \mathrm{~m} / \mathrm{min}$ for the first $5 \mathrm{~min}, 7 \mathrm{~m} / \mathrm{min}$ for the next $5 \mathrm{~min}$ and then $10 \mathrm{~m} / \mathrm{min}$ for the last $20 \mathrm{~min}$ with $0^{\circ}$ inclination. The animals in the SD group were placed on the treadmill for $30 \mathrm{~min}$, without being induced to run.

Tissue processing for histology. Tissue processing was performed according to a previously published procedure (20). Briefly, animals ( $n=7 /$ group) were anesthetized with sodium pentobarbital (40 mg/kg, i.p.; JW Pharmaceutical Co., Ltd., Seoul, Korea) and perfused transcardially with $4 \%$ paraformaldehyde. Brain tissues were serially sectioned into $30 \mu \mathrm{m}$ coronal sections.

Cresyl violet $(\mathrm{CV})$ staining. To investigate morphological alterations, CV staining was performed according to a previously published procedure (24). Briefly, the sections were stained with $1 \% \mathrm{CV}$ acetate (Sigma-Aldrich; Merck KGaA, Darmstadt, Germany) and immersed in serial ethanol baths. CV-stained structures were observed under an AxioM1 light microscope (Zeiss AG, Oberkochen, Germany) equipped with a camera (Axiocam; Zeiss AG) and photomicrographs were captured. The CV-stained structures were examined in a $250 \times 250 \mu \mathrm{m}$ area that included the stratum pyramidale at the center of the hippocampal CA1 region, or in the whole dentate gyrus, using the image analysis system Optimas version 6.5 (CyberMetrics, Scottsdale, AZ, USA).

Fluoro-Jade B (F-J B) histofluorescence staining. Histofluorescence staining was performed according to a previously published procedure (25). F-J B (high-affinity fluorescent marker for the localization of neuronal degeneration) histofluorescence staining was performed to examine neuronal degeneration. Briefly, the sections were immersed in a solution containing $1 \%$ sodium hydroxide in $80 \%$ alcohol, transferred to a solution containing $0.06 \%$ potassium permanganate diluted in water, then transferred to an aquaeous solution containing $0.0004 \%$ F-J B (Histo-Chem, Inc., Jefferson, AR, USA). After washing 3 times in water, the sections were placed on a slide warmer $\left(\sim 50^{\circ} \mathrm{C}\right)$ and examined using an epifluorescent microscope (Zeiss AG) with blue (450-490 nm) excitation source and a barrier filter.

Immunohistochemistry. Immunohistochemistry was performed according to our previously published procedure (24). Briefly, immunostaining was performed using mouse anti-glial fibrillary acidic protein (GFAP; 1:800; cat no. MAB360; EMD Millipore, Billerica, MA, USA) for astrocytes and rabbit anti-ionized calcium binding adaptor molecule 1 (Iba-1; 1:800; cat no. 019-19741; Wako Pure Chemical Industries, Ltd., Osaka, Japan) for microglia overnight at $4^{\circ} \mathrm{C}$. Subsequently, samples were incubated with biotinylated horse anti-mouse immunoglobulin G (1:250; cat no. BA2000; Vector Laboratories, Inc., Burlingame, CA, USA) or goat anti-rabbit antibodies (1:250; cat no. BA1000; Vector Laboratories, Inc.) for $2 \mathrm{~h}$ at room temperature, and streptavidin peroxidase complex (1:200; Vector Laboratories, Inc.) for $1 \mathrm{~h}$ at room temperature. To establish the specificity of the 


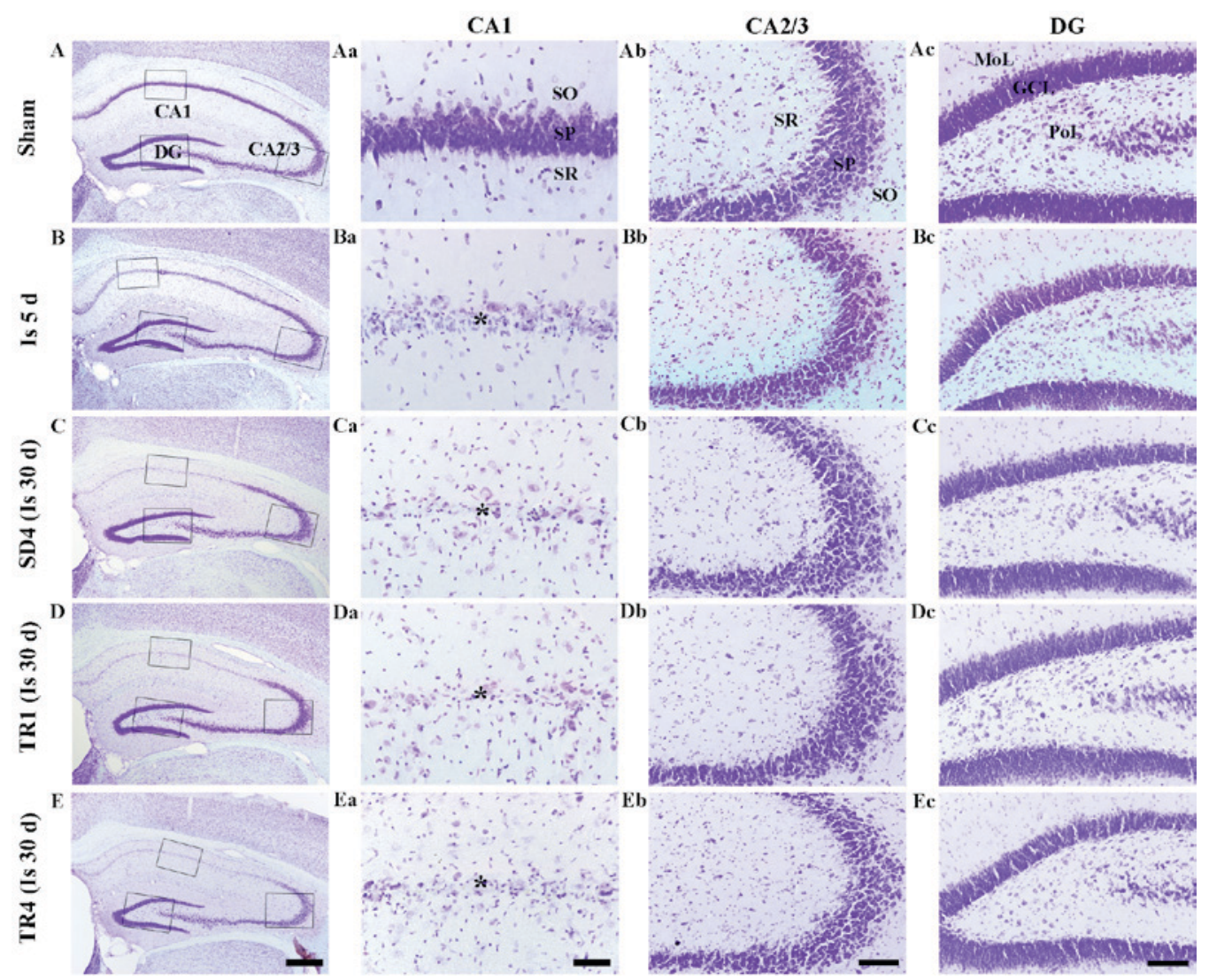

Figure 1. CV staining in the (A) sham, (B and C) ischemia and (Da and E) TR groups. Only a small number of CV-positive cells were detected in the SP (asterisk) of the CA1 region 5 days post-ischemia. In the SD4, TR1 and TR4 groups, the pattern of CV-positive cells distribution was similar to the ischemia group at 5 days post-ischemia. Scale bar: (Aa-Ea) $400 \mu \mathrm{m}$, (Ab-Eb) $40 \mu \mathrm{m}$ and (Ac-Ec and Ad-Ed) $100 \mu \mathrm{m}$. CV, cresyl violet; TR, treadmill exercise; SD, sedentary routine; DG, dentate gyrus; GCL, granule cell layer; MoL, molecular layer; PoL, polymorphic layer; SO, stratum oriens; SR, stratum radiatum; SP, stratum pyramidale.

immunostaining, a negative control test was performed and resulted in the absence of immunoreactivity in all structures.

Data analysis. In order to quantitatively analyze the number of F-J B-positive cells, digital images from seven sections per animal were captured using a light microscope (AxioM1; Zeiss AG) equipped with a digital camera (Axiocam; Zeiss AG) and connected to a PC monitor. The number of F-J B-positive cells was counted in a $250 \times 250 \mu \mathrm{m}$ square including the stratum pyramidale at the center of the hippocampal CA1 region or in the whole dentate gyrus using the image analysis system Optimas version 6.5 (CyberMetrics). Cell counts were carried out by averaging the counts from each animal.

To quantitatively analyze the density of GFAP- and Iba-1-immunoreactive structures, the corresponding hippocampal areas were measured from seven sections per animal. Images of all GFAP- and Iba-1-immunoreactive structures were captured through an AxioM1 light microscope (Zeiss AG) equipped with a camera (Axiocam; Zeiss AG) and connected to a PC monitor. Densities of GFAP- and Iba-1-immunoreactive structures were evaluated on the basis of optical density (OD), obtained following the transformation of the mean gray level using the formula: OD=log (256/mean gray level). The background was subtracted and the OD ratio for each image was calibrated as \% relative optical density (ROD) using Adobe Photoshop version 8.0 (Adobe Systems, San Jose, CA, USA) and ImageJ software version 1.49 (National Institutes of Health, Bethesda, MD, USA). The mean value of the OD of the sham group was designated as $100 \%$ and the ROD in each group was calibrated and expressed as a percentage of the sham group.

Statistical analysis. Data are expressed as the mean \pm standard error of the mean of at least 2 independent experiments. Data from F-J B immunofluorescence and immunohistochemical staining were analyzed using one-way analysis of variance, followed by a post hoc Bonferroni-Dunn Test using SPSS version 17.0 (SPSS, Inc., Chicago, IL, USA). P<0.05 was considered to indicate a statistically significant difference.

\section{Results}

\section{CV-positive cells}

Sham group. CV staining is presented in Fig. 1. CV-positive cells were detected throughout the hippocampus; in particular, they were aggregated in the stratum pyramidale of the hippocampus proper (CA1-3 regions) and the granular cell layer of the dentate gyrus (Fig. 1Aa-d).

Ischemia groups. CV-positive cells were markedly decreased in the CA1 stratum pyramidale, but not in the other subregions, 5 days post-ischemia (Fig. $1 \mathrm{Ba}-\mathrm{d}$ ). In the SD4 group, the distribution pattern of CV-positive cells was similar to the ischemia group at 5 days post-ischemia (Fig. 1Ca-d). 


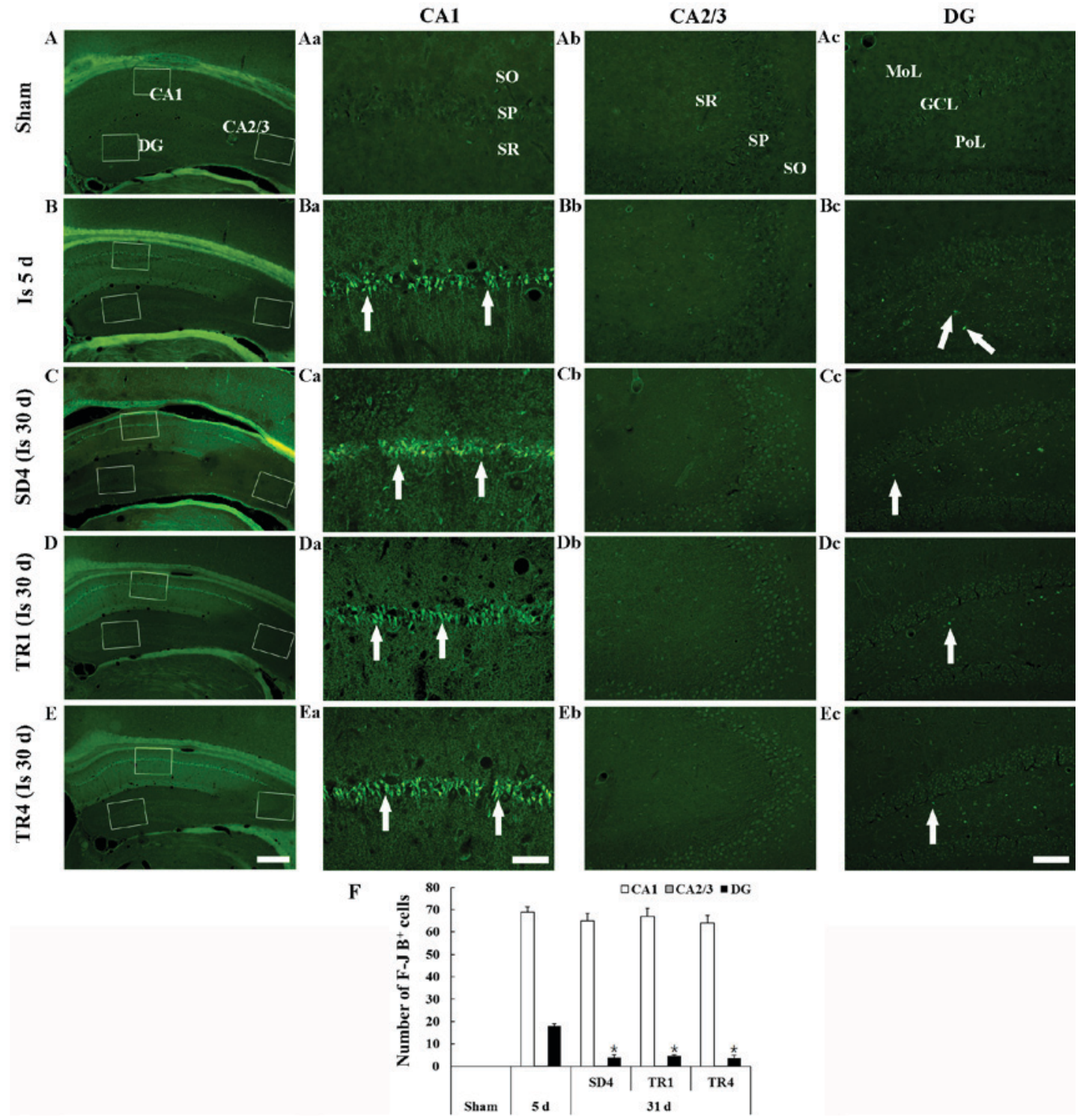

Figure 2. F-J B histofluorescence staining in the (A) sham, (B and C) ischemia and (D and E) TR groups. In the sham group, no F-J B-positive cells were detected. However, at 5 days post-ischemia, numerous F-J B-positive neurons (arrows) were detected in the SP of the CA1 region and in the PoL of the DG. In the SD4, TR1 and TR4 groups, the number of F-J B-positive neurons (arrows) in the SP of the CA1 region and in the PoL of the DG was similar between the groups. Scale bar: (Aa-Ea) $400 \mu \mathrm{m}$, (Ab-Eb) $40 \mu \mathrm{m}$ and (Ac-Ec and Ad-Ed) $100 \mu \mathrm{m}$. (F) Number of F-J B-positive cells in the SP of the CA1 and CA2/3 regions and in the PoL of the $\mathrm{DG}$ (n=7/group). Data are presented as the mean \pm standard error of mean. ${ }^{*} \mathrm{P}<0.05$ vs. ischemia group at 5 days post-ischemia F-J B, Fluoro-Jade B; TR, treadmill exercise; SD, sedentary routine; DG, dentate gyrus; GCL, granule cell layer; MoL, molecular layer; PoL, polymorphic layer; SO, stratum oriens; SR, stratum radiatum; SP, stratum pyramidale.

TR-groups. In the TR1 and TR4 groups, the distribution pattern of CV-positive cells in the hippocampus was also similar to the SD4 group (Fig. 1Da-d and Ea-d).

\section{F-J B-positive cells}

Sham group. F-J B staining is presented in Fig. 2. F-J B-positive cells were not detected in any layers of the hippocampus proper and the dentate gyrus (Fig. 2Aa-d).

Ischemia groups. A total of 5 days post-ischemia, numerous F-J B-positive cells were detected in the stratum pyramidale of the CA1 region and a small number of F-J B-positive cells were detected in the polymorphic layer of the dentate gyrus (Fig. 2Ba-d and F). In the SD4 group, the distribution pattern and number of F-J B-positive cells in the CA1 stratum pyramidale was similar to the ischemia-group at 5 days post-ischemia; however, the number of F-J B-positive cells was significantly decreased in the polymorphic layer of the dentate gyrus (Fig. 2Ca-d and F).

TR-groups. In the TR1 and TR4 groups, the number of F-J B-positive cells in the CA1 stratum pyramidale and in the polymorphic layer of the dentate gyrus was similar to the SD4 group and no significant difference in the number of F-J B-positive cells was observed between the TR1 and TR4 groups (Fig. 2Da-d, Ea-d and F).

\section{GFAP-immunoreactive astrocytes}

Sham group. GFAP staining is presented in Fig. 3. GFAPimmunoreactive astrocytes in the sham group were easily detected in all layers of the hippocampus proper and the dentate gyrus. The astrocytes appeared to be at resting 


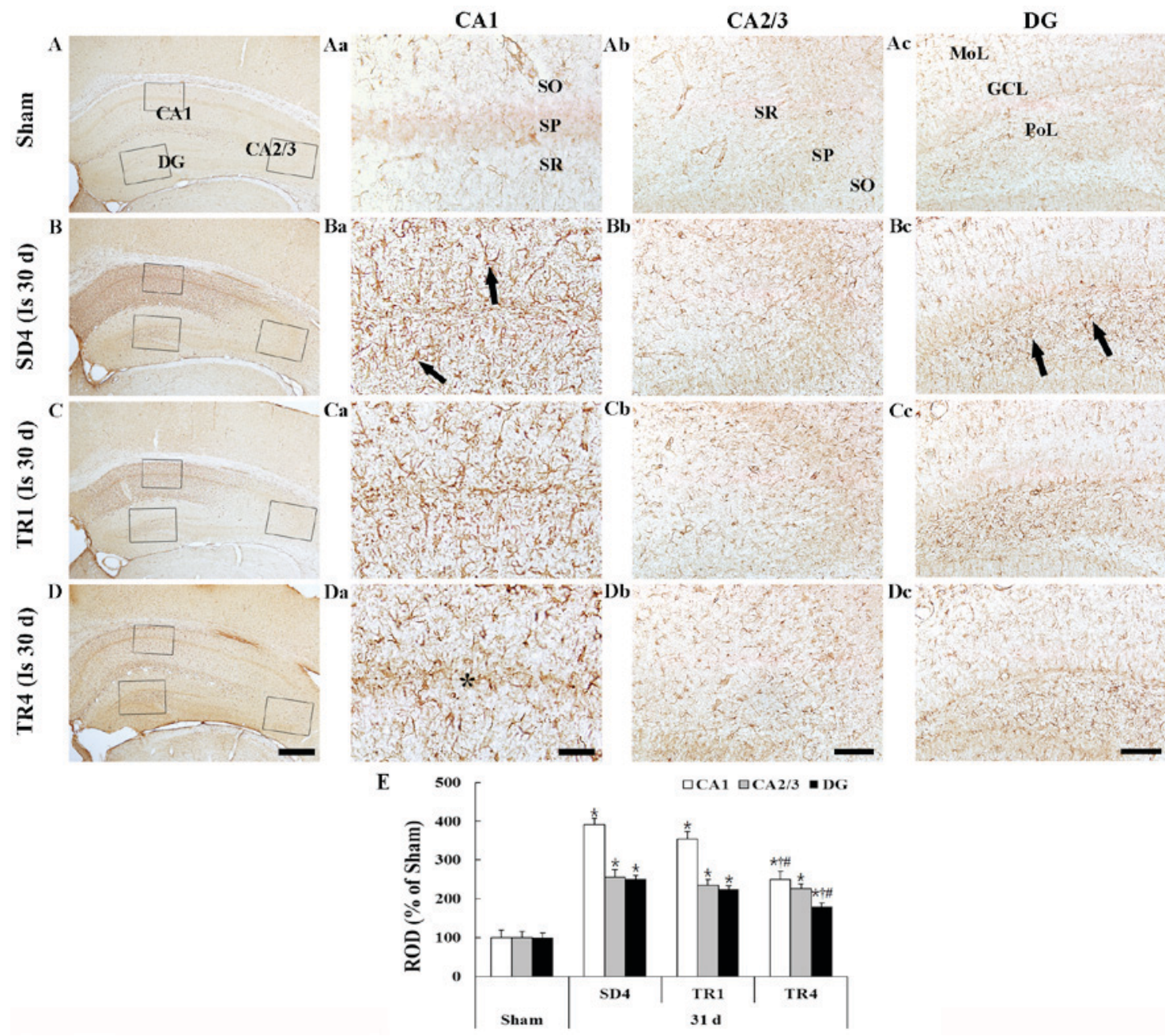

Figure 3. GFAP immunohistochemistry in the (A) sham, (B) SD4, (C) TR1 and (D) TR4 groups. In the SD4 group, GFAP-immunoreactive astrocytes (arrows) were activated in the CA1 region and in the PoL of the DG. In the TR1 group, the activation was similar to the SD4 group; however, in the TR4 group, the activation was significantly decreased compared with the SD4 group. Scale bar: (Aa-Da) $400 \mu \mathrm{m}$, (Ab-Db) $40 \mu \mathrm{m}$ and (Ac-Dc and Ad-Dd) $100 \mu \mathrm{m}$. (E) ROD expressed as a percentage of GFAP-immunoreactive structures ( $\mathrm{n}=7$ /group). Data are presented as the mean \pm standard error of mean. " $\mathrm{P}<0.05$ vs. sham group; ${ }^{\dagger} \mathrm{P}<0.05$ vs. SD4 group; ${ }^{\#} \mathrm{P}<0.05$ vs. TR1 group. GFAP, glial fibrillary acidic protein; SD, sedentary routine; TR, treadmill exercise; DG, dentate gyrus; GCL, granule cell layer; MoL, molecular layer; PoL, polymorphic layer; SO, stratum oriens; SP, stratum pyramidale; SR, stratum radiatum; ROD, relative optical density.

form and had a small body with thread-like thin processes (Fig. 3Aa-d).

Ischemia group. In the SD4 group, numerous GFAPimmunoreactive astrocytes demonstrated a typical activated form that had a punctuated cytosol with thick processes (Fig. 3Ba-d). The density of the GFAP-immunoreactive structures (ROD) was significantly increased in all subregions compared with in the sham group $(\mathrm{P}<0.05$; Fig. $3 \mathrm{E})$; in particular, the activation was marked in the CA1 region and in the polymorphic layer of the dentate gyrus.

TR groups. In the TR1 group, the morphology of GFAPimmunoreactive astrocytes in the hippocampus proper and the dentate gyrus was similar to the SD4 group (Fig. 3Ca-d) and the ROD of GFAP-immunoreactive structures was not significantly different compared with the SD4 group (Fig. 3E). However, in the TR4 group the ROD was significantly decreased $(\mathrm{P}<0.05)$ compared with in the SD4 and TR1 groups (Fig. 3Da-d and E).

\section{Iba-1-immunoreactive microglia}

Sham group. Iba-1 staining is presented in Fig. 4. Iba-1immunoreactive microglia were evenly distributed throughout the hippocampus. The microglia appeared to be at resting form and exhibited fine processes with web-like network characteristics (Fig. 4Aa-d).

Ischemia group. In the SD4 group, Iba-1-immunoreactive microglia were markedly altered in the CA1 region and in the polymorphic layer of the dentate gyrus; they exhibited bulky cytoplasm with short and thickened processes, which represents the activated form (Fig. 4Ba-d and E). In particular, activated Iba-1-immunoreactive microglia were aggregated in the stratum pyramidale of the CA1 region. The ROD of the Iba-1-immunoreactive structures was significantly increased $(\mathrm{P}<0.05)$ in the CA1 region and the polymorphic layer of the dentate gyrus compared with in the sham group (Fig. 4E).

$T R$ groups. In the TR 1 group, the distribution pattern of Iba-1-immunoreactive microglia in the hippocampus 


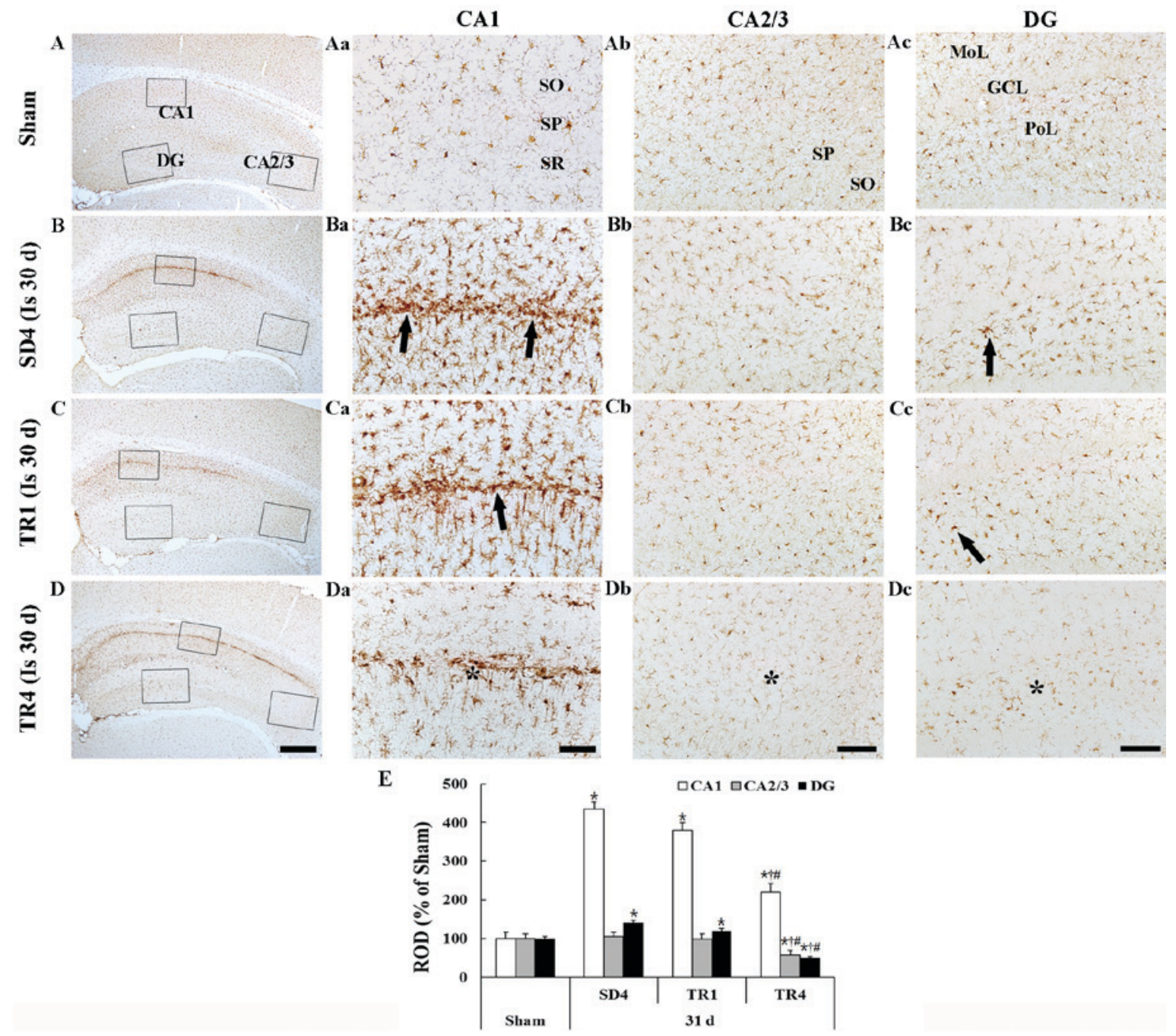

Figure 4. Iba-1 immunohistochemistry in the (A) sham, (B) SD4, (C) TR1 and (D) TR4 groups. Iba-1-immunoreactive microglia were activated (arrows) in the CA1 region and the PoL of the DG in the SD4 group. In the TR4 group, the activation of Iba-1-immunoreactive microglia was significantly decreased (asterisks) although the activation in the TR1 group was similar to the SD4 group. Scale bar: (Aa-Da) $400 \mu \mathrm{m}$, (Ab-Db) $40 \mu \mathrm{m}$ and (Ac-Dc and Ad-Dd) $100 \mu \mathrm{m}$. (E) ROD expressed as a percentage of Iba-1 immunoreactive structures ( $\mathrm{n}=7 /$ group). Data are presented as the mean \pm standard error of the mean. ${ }^{*} \mathrm{P}<0.05$ vs. the sham group; ${ }^{\mathrm{P}} \mathrm{P}<0.05$ vs. the $\mathrm{SD} 4$ group; ${ }^{\mathrm{P}} \mathrm{P}<0.05$ vs. the TR1 group). Iba- 1 , ionized calcium binding adaptor molecule $1 ; \mathrm{SD}$, sedentary routine; TR, treadmill exercise; DG, dentate gyrus; GCL, granule cell layer; MoL, molecular layer; SO, stratum oriens; SP, stratum pyramidale; SR, stratum radiatum; ROD, relative optical density.

was similar to the SD4 group; however, the activation of Iba-1-immunoreactive microglia was slightly decreased in the CA1 region and the dentate gyrus (Fig. 4Ca-d). In the TR4 group, the ROD of activated Iba-1-immunoreactive microglia was significantly decreased $(\mathrm{P}<0.05)$ in the $\mathrm{CA} 1$ region and the dentate gyrus compared with in the SD4 and TR1 groups (Fig. 4Da-c and E).

\section{Discussion}

Ischemic brain damage can lead to the development of neuronal damage and gliosis (26), and result in long-term functional disability $(27,28)$. The present study investigated the effects of long- and short-term post-ischemic treadmill exercise on neuronal death and glial activation in the aged gerbil hippocampus induced by 5 min of transient cerebral ischemia.

In the present study, at 5 days post-ischemia, a distinct neuronal loss was observed in the CA1 stratum pyramidale and in the polymorphic layer of the dentate gyrus in the aged gerbil hippocampus, as determined using CV and F-J B staining. This result is consistent with our previous findings, which demonstrated that a significant neuronal loss in the aged gerbil hippocampus was detected in the CA1 stratum pyramidale (5) and in the polymorphic layer of the dentate gyrus (29) 5 days after transient ischemia. At 31 days post-ischemia in the SD4 group, the number of F-J B-positive cells (dead neurons) in the CA1 region was similar to that at 5 days post-ischemia. Furthermore, the present study is the first, to the best of our knowledge, to report that short- and long-term post-ischemic treadmill exercise did not exhibit any neuroprotection in the TR1 and TR4 groups; the numbers of F-J B-positive neurons in the CA1 region and the dentate gyrus were no different compared with the SD4 group. It has previously been reported that short- and long-term treadmill exercise, initiated prior to ischemic neuronal death, exerted a neuroprotective effect by suppressing transient cerebral ischemia-induced apoptosis of the neurons in the CA1 region (21-23). Based on the findings 
of the present study and previous studies, it may be concluded that treadmill exercise begun after transient cerebral ischemia-induced neuronal degeneration cannot protect neurons in the aged hippocampus.

In the present study, the significant activation of GFAP-immunoreactive astrocytes and Iba-1-immunoreactive microglia was observed in the CA1 region and the dentate gyrus of the SD4 group, and their ROD was significantly increased compared with in the sham group. However, 4 weeks of post-ischemic treadmill exercise significantly reduced the number of activated astrocytes and microglia in the CA1 region and in the dentate gyrus compared with the sedentary control (SD4 group). Conversely, 1 week of treadmill exercise did not effectively decrease their activation in the ischemic hippocampus. It is well known that ischemic hippocampus pathology is closely associated with an acute and prolonged inflammatory response, which is characterized by the production of inflammatory cytokines and the activation of resident glial cells $(30,31)$. In this regard, previous studies have demonstrated that wheel-running exercise attenuated age-related astrocyte hypertrophy (32) and microglial proliferation (33). In addition, chronic exercise inhibited the activation of astrocytes and microglia, and other inflammatory-related factors, including inducible nitric oxide synthase, in murine models of Alzheimer's and Parkinson's diseases $(34,35)$. The present results, along with the aforementioned findings, indicated that long-term treadmill exercise may alleviate increased neuroinflammation in the aged gerbil hippocampus induced by transient cerebral ischemia.

In conclusion, the present study suggested that 4 weeks of treadmill exercise, initiated after neuronal death, cannot influence neuronal protection; however, the exercise can effectively alleviate transient cerebral ischemia-induced gliosis in the hippocampus of aged gerbils.

\section{Acknowledgements}

The present study was supported by grants from the Osong Innovation Center funded by the Ministry of Health \& Welfare, Republic of Korea (grant no. HO14C0001) and the Basic Science Research Program through the National Research Foundation of Korea (NRF) funded by the Ministry of Education (grant no. NRF-2014R1A1A3051721).

\section{References}

1. Kirino T: Delayed neuronal death in the gerbil hippocampus following ischemia. Brain Res 239: 57-69, 1982.

2. Lin CS, Polsky K, Nadler JV and Crain BJ: Selective neocortical and thalamic cell death in the gerbil after transient ischemia. Neuroscience 35: 289-299, 1990.

3. Petito CK, Torres-Munoz J, Roberts B, Olarte JP, Nowak TS Jr and Pulsinelli WA: DNA fragmentation follows delayed neuronal death in CA1 neurons exposed to transient global ischemia in the rat. J Cereb Blood Flow Metab 17: 967-976, 1997.

4. Horn M and Schlote W: Delayed neuronal death and delayed neuronal recovery in the human brain following global ischemia. Acta Neuropathol 85: 79-87, 1992.

5. Lee CH, Yoo KY, Choi JH, Park OK, Hwang IK, Kim SK, Kang IJ, Kim YM and Won MH: Neuronal damage is much delayed and microgliosis is more severe in the aged hippocampus induced by transient cerebral ischemia compared to the adult hippocampus. J Neurol Sci 294: 1-6, 2010.
6. Tamagaki C, Murata A, Asai S, Takase K, Gonno K, Sakata T and Kinoshita T: Age-related changes of cornu ammonis 1 pyramidal neurons in gerbil transient ischemia. Neuropathology 20: 221-227, 2000

7. Bernal GM and Peterson DA: Phenotypic and gene expression modification with normal brain aging in GFAP-positive astrocytes and neural stem cells. Aging cell 10: 466-482, 2011.

8. Gehrmann J, Bonnekoh P, Miyazawa T, Hossmann KA and Kreutzberg GW: Immunocytochemical study of an early microglial activation in ischemia. J Cereb Blood Flow Metab 12: 257-269, 1992.

9. Norden DM and Godbout JP: Review: Microglia of the aged brain: Primed to be activated and resistant to regulation. Neuropathol Appl Neurobiol 39: 19-34, 2013.

10. Choi JH and Won MH: Microglia in the normally aged hippocampus. Lab Anim Res 27: 181-187, 2011.

11. Nedergaard M and Dirnagl U: Role of glial cells in cerebral ischemia. Glia 50: 281-286, 2005.

12. Lai AY and Todd KG: Microglia in cerebral ischemia: Molecular actions and interactions. Can J Physiol Pharmacol 84: 49-59, 2006.

13. Kwakkel G, van Peppen R, Wagenaar RC, Wood Dauphinee S, Richards C, Ashburn A, Miller K, Lincoln N, Partridge C, Wellwood I and Langhorne P: Effects of augmented exercise therapy time after stroke: a meta-analysis. Stroke 35: 2529-2539, 2004.

14. Zhang P, Zhang Q, Pu H, Wu Y, Bai Y, Vosler PS, Chen J, Shi H, Gao Y and Hu Y: Very early-initiated physical rehabilitation protects against ischemic brain injury. Front Biosci (Elite Ed) 4: 2476-2489, 2012

15. Chen MF, Huang TY, Kuo YM, Yu L, Chen HI and Jen CJ: Early postinjury exercise reverses memory deficits and retards the progression of closed-head injury in mice. J Physiol 591: 985-1000, 2013.

16. Fang KM, Cheng FC, Huang YL, Chung SY, Jian ZY and Lin MC: Trace element, antioxidant activity, and lipid peroxidation levels in brain cortex of gerbils after cerebral ischemic injury. Biol Trace Elem Res 152: 66-74, 2013.

17. Liu YR, Lei RY, Wang CE, Zhang BA, Lu H, Zhu HC and Zhang GB: Effects of catalpol on ATPase and amino acids in gerbils with cerebral ischemia/reperfusion injury. Neurol Sci 35: 1229-1233, 2014

18. Shcherbak NS, Galagudza MM, Ovchinnikov DA, Kuzmenkov AN, Yukina GY, Barantsevich ER, Tomson VV and Shlyakhto EV: Activity of succinate dehydrogenase in the neocortex and hippocampus of Mongolian gerbils with ischemic and reperfusion brain injury. Bull Exp Biol Med 155: 14-17, 2013.

19. Wang W, Wang T, Feng WY, Wang ZY, Cheng MS and Wang YJ: Ecdysterone protects gerbil brain from temporal global cerebral ischemia/reperfusion injury via preventing neuron apoptosis and deactivating astrocytes and microglia cells. Neurosci Res 81-82: 21-29, 2014.

20. Ahn JH, Choi JH, Park JH, Kim IH, Cho JH, Lee JC, Koo HM, Hwangbo $\mathrm{G}$, Yoo KY, Lee $\mathrm{CH}$, et al: Long-term exercise improves memory deficits via restoration of myelin and microvessel damage, and enhancement of neurogenesis in the aged gerbil hippocampus after ischemic stroke. Neurorehabil Neural Repair 30: 894-905, 2016.

21. Sim YJ, Kim H, Kim JY, Yoon SJ, Kim SS, Chang HK, Lee TH, Lee HH, Shin MC, Shin MS and Kim CJ: Long-term treadmill exercise overcomes ischemia-induced apoptotic neuronal cell death in gerbils. Physiol Behav 84: 733-738, 2005.

22. Sim YJ, Kim SS, Kim JY, Shin MS and Kim CJ: Treadmill exercise improves short-term memory by suppressing ischemia-induced apoptosis of neuronal cells in gerbils. Neurosci Lett 372: 256-261, 2004.

23. Lee MH, Kim H, Kim SS, Lee TH, Lim BV, Chang HK, Jang MH, Shin MC, Shin MS and Kim CJ: Treadmill exercise suppresses ischemia-induced increment in apoptosis and cell proliferation in hippocampal dentate gyrus of gerbils. Life Sci 73: 2455-2465, 2003.

24. Ahn JH, Choi JH, Kim JS, Lee HJ, Lee CH, Yoo KY, Hwang IK, Lee YL, Shin HC and Won MH: Comparison of immunoreactivities in 4-HNE and superoxide dismutases in the cervical and the lumbar spinal cord between adult and aged dogs. Exp Gerontol 46: 703-708, 2011.

25. Candelario-Jalil E, Alvarez D, Merino N and León OS: Delayed treatment with nimesulide reduces measures of oxidative stress following global ischemic brain injury in gerbils. Neurosci Res 47: 245-253, 2003. 
26. Sugawara T, Lewén A, Noshita N, Gasche Y and Chan PH: Effects of global ischemia duration on neuronal, astroglial, oligodendroglial, and microglial reactions in the vulnerable hippocampal CA1 subregion in rats. J Neurotrauma 19: 85-98, 2002.

27. Li DQ, Bao YM, Li Y, Wang CF, Liu Y and An LJ: Catalpol modulates the expressions of Bcl-2 and Bax and attenuates apoptosis in gerbils after ischemic injury. Brain Res 1115: 179-185, 2006.

28. Takagi N: Pathology and strategies for the treatment of ischemic brain injury. Yakugaku Zasshi 129: 1215-1219, 2009 (In Japanese).

29. Ahn JH, Shin BN, Park JH, Kim IH, Cho JH, Chen B, Lee TK, Tae HJ, Lee JC, Cho JH, et al: Long-term observation of neuronal degeneration and microgliosis in the gerbil dentate gyrus after transient cerebral ischemia. J Neurol Sci 363: 21-26, 2016.

30. Danton GH and Dietrich WD: Inflammatory mechanisms after ischemia and stroke. J Neuropathol Exp Neurol 62: 127-136, 2003.
31. Stoll G and Jander S: The role of microglia and macrophages in the pathophysiology of the CNS. Prog Neurobiol 58: 233-247, 1999.

32. Latimer CS, Searcy JL, Bridges MT, Brewer LD, Popović J, Blalock EM, Landfield PW, Thibault O and Porter NM: Reversal of glial and neurovascular markers of unhealthy brain aging by exercise in middle-aged female mice. PLoS One 6: e26812, 2011.

33. Kohman RA, DeYoung EK, Bhattacharya TK, Peterson LN and Rhodes JS: Wheel running attenuates microglia proliferation and increases expression of a proneurogenic phenotype in the hippocampus of aged mice. Brain Behav Immun 26: 803-810, 2012.

34. Sung YH, Kim SC, Hong HP, Park CY, Shin MS, Kim CJ, Seo JH, Kim DY, Kim DJ and Cho HJ: Treadmill exercise ameliorates dopaminergic neuronal loss through suppressing microglial activation in Parkinson's disease mice. Life Sci 91: 1309-1316, 2012.

35. Leem YH, Lee YI, Son HJ and Lee SH: Chronic exercise ameliorates the neuroinflammation in mice carrying NSE/htau23. Biochem Biophys Res Commun 406: 359-365, 2011. 